\title{
Correction to: Depressive symptom trajectories in the first 10 years of diabetes diagnosis: antecedent factors and link with future disability in Taiwan
}

\author{
Ching-Ju Chiu ${ }^{1} \cdot$ Yu-Hsuan Tseng ${ }^{2} \cdot$ Yu-Ching Hsu ${ }^{3} \cdot$ Shang-Te Wu ${ }^{4}$ Linda A. Wray ${ }^{5}$
}

Published online: 9 June 2018

๑) Springer-Verlag GmbH Germany, part of Springer Nature 2018

\section{Correction to: \\ Soc Psychiatry Psychiatr Epidemiol (2017) 52:829-836 \\ https://doi.org/10.1007/s00127-016-1314-4}

The original publication of this article contained an error in the list of the authors, in which our last contributing author, Dr. Linda A. Wray was missing. This error is corrected via this correction.

The original article can be found online at https://doi.org/10.1007/ s00127-016-1314-4.

Ching-Ju Chiu

cjchiu@mail.ncku.edu.tw

1 Institute of Gerontology, College of Medicine, National Cheng Kung University, No. 1, University Road,

Tainan 70101, Taiwan

2 Department of Occupational Therapy, College of Medicine, National Cheng Kung University, Tainan 70101, Taiwan

3 Department of Chinese Medicine, Tainan Hospital, Ministry of Health and Welfare, Tainan 70043, Taiwan

4 Department of Neurology, Kao General Hospital, Tainan 70054, Taiwan

5 Department of Biobehavioral Health, The Pennsylvania State University, University Park, PA, USA 